\title{
NEW OPERATIVE METHOD FOR ACUTE TYPE B DISSECTION: LETT CAROTID ARTERY-LEFT SUBCLAVIAN ARTERY BYPASS COMBINED WITH ENDOVASCULAR STENT-GRAFT IMPLANTATION
}

Norihisa Shigemura, MD, Masaaki Kato, MD, Toru Kuratani, MD, Yasunobu Funakoshi, MD, and Mitsunori Kaneko, MD, Osaka, Japan

From the Division of Cardiovascular Surgery, Osaka Prefectural Hospital, Sumiyoshiku Osaka, Japan.

Received for publication Nov 30, 1999; accepted for publication March 20, 2000.

Address for reprints: Norihisa Shigemura, MD, Division of Cardiovascular Surgery, Osaka Prefectural Hospital, 3-1-56 Mandai-higashi, Sumiyoshiku Osaka 558-8558, Japan.

J Thorac Cardiovasc Surg 2000;120;406-8

Copyright (C) 2000 by The American Association for Thoracic Surgery

$0022-5223 / 2000 \$ 12.00+0 \quad \mathbf{1 2 / 5 4 / 1 0 7 2 7 9}$

doi: $10.1067 / \mathrm{mtc} .2000 .107279$
For several years, the newest treatment for entry-site closure in type B aortic dissection has been stent-graft implantation. ${ }^{1-3}$ However, in the case of entry sites located near the origin of the left subclavian artery, persistent perigraft leakage often results because the stent-graft is not supported by a sufficient uncurved area of aorta to allow complete attachment to the intima of the true lumen. In response, we have applied a transcatheter stent-graft implantation method, which we combine with a bypass between the left carotid and the left subclavian arteries. The following case history presents an application of this approach. 

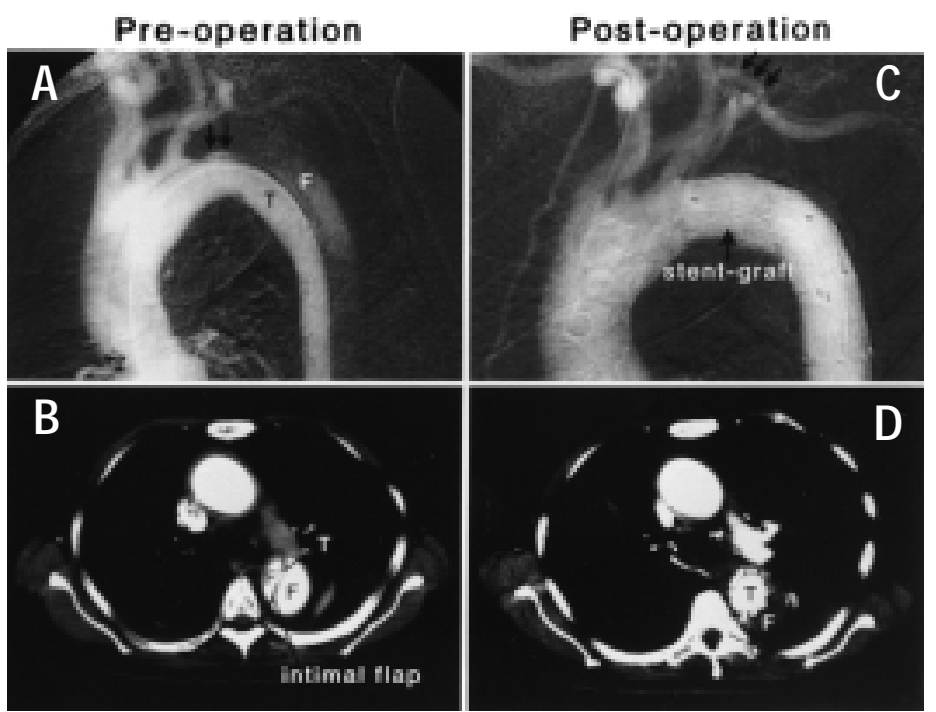

Fig 1. Preoperative (A and $\mathbf{B})$ and postoperative $(\mathbf{C}$ and $\mathbf{D})$ aortograms and computed tomograms. A, Preoperative aortogram shows thoracic aortic dissection ( $T$, true lumen; $F$, false lumen) with an entry site below the left subclavian artery (arrows). B, Preoperative computed tomogram shows an intimal flap in the descending thoracic aorta. C, Postoperative aortogram reveals that the entry was closed by the endovascular stent-graft (large arrow) and that the bypass between the left carotid artery and left subclavian artery was patent (small arrows). D, Postoperative computed tomogram demonstrates complete exclusion of the false lumen through the descending aorta.

Clinical summary. A 57-year-old woman with sharp chest and back pain was sent to our hospital on an emergency basis with suspected aortic dissection. An enhanced computed tomographic scan of the chest and abdomen and aortography showed an intimal flap from the distal aortic arch to the end of the abdominal aorta, compatible with Stanford type B aortic dissection (Fig 1, A and B). The entry site was located at the distal aortic arch, just $2.5 \mathrm{~cm}$ beyond the origin of the left subclavian artery. The true lumen was narrowed by compression from the false lumen, which was enlarged in the descending aorta. In the aortogram the main abdominal aortic branches, except the right renal artery, projected in the true lumen; the right renal artery was obscured by compression from the false lumen. Medical hypotensive therapy was started soon after admission, but we decided to treat the patient surgically during the acute phase because the aortic diameter was large $(\geq 40 \mathrm{~mm})$ and the aneurysm was forecast to enlarge during the chronic phase. ${ }^{4}$ We closed the entry site with a stent-graft on the seventh day after the onset of dissection.

With the patient under general anesthesia, a 6-cm transverse incision was made above the left clavicle, and the bypass was constructed between the left carotid artery and the left subclavian artery with a polytetrafluoroethylene graft ${ }^{*}(8$

*Gore-Tex graft; registered trademark of W. L. Gore \& Associates, Inc, Flagstaff, Ariz. mm) (Fig 2, B). This bypass graft made it possible to close the orifice of the left subclavian artery and to secure a sufficient area of attachment between the stent-graft and the intima of the true lumen. In the meantime, a femoral artery and vein and a brachial artery and vein were secured for use in the implantation of the stent-graft. A specially devised stentgraft, constructed in our institution and composed of a thinwalled woven Dacron graft (Nakao Filter Media Corp, Osaka, Japan) and a Gianturco stent (GZV40-50, GZV40-75, William Cook Europe A/S, Bjaeverskov, Denmark), was fluoroscopically deployed via a $20 \mathrm{~F}$ sheath through the femoral artery (Fig 2,C). At deployment, both the superior and inferior venae cavae were occluded with balloons from the brachial vein and the femoral vein, and the systolic blood pressure was lowered to $50 \mathrm{~mm} \mathrm{Hg}$. Aortography confirmed complete closure of the entry site. No complication was observed after the operation. Postoperative aortography revealed that the narrowing of the true lumen by compression from the false lumen had been improved and the right renal artery was clearly projected, and computed tomography showed complete clot formation in the excluded false lumen (Fig 1, $C$ and $D$ ). The patient was discharged from the hospital on the 14th day after the operation.

Comment. In many institutions, medical hypotensive therapy has been the preferred treatment for the patient with acute type B dissection. However, $20 \%$ to $40 \%$ of patients who have passed through the acute phase with medical hypotensive therapy probably need surgery during the chronic phase because of 


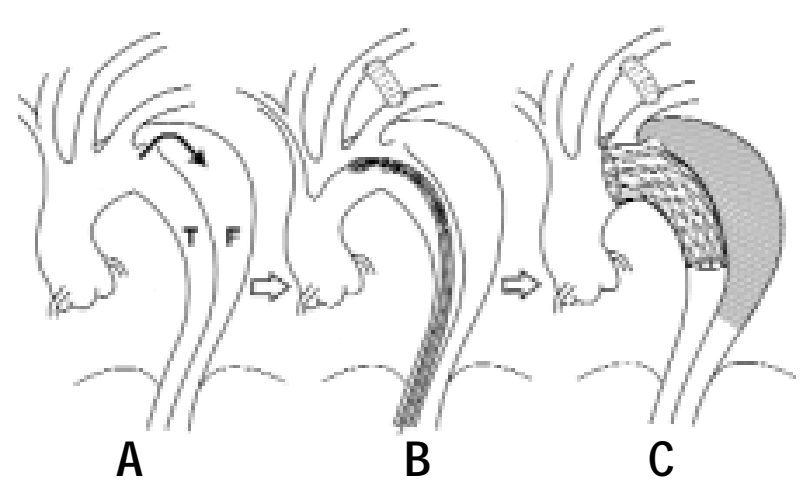

Fig 2. Schema of the new stent-graft implantation method for type B aortic dissection. A, The entry site of type B dissection is located near the origin of the left subclavian artery. B, After the bypass between the left carotid and left subclavian arteries is completed, a $20 \mathrm{~F}$ catheter sheath is inserted via the femoral artery and the stent-graft is delivered to the intended position by the pushing rod. $\mathbf{C}$, The stent-graft is deployed, extending from the distal arch to the descending aorta, closing the origin of the left subclavian artery and permitting the false lumen to clot.

enlargement of the aortic dissection. It is important to give surgical treatment to patients who have a large aortic diameter and a patent primary entry site in the thoracic aorta ${ }^{4}$ and to close the entry site in the acute phase, when it is easier for the false lumen to shrink. ${ }^{1}$ However, in those cases in which the entry site is near the origin of the left subclavian artery, it is impossible to close the entry site completely because the transcatheter approach via the femoral artery with the current devices provides insufficient attachment on the proximal side. In our experience, complete closure of the entry site requires that the distance between the left subclavian artery and the entry site be more than $2.5 \mathrm{~cm}$ and the attachment area be straight. The left carotid artery-left subclavian artery bypass method, which has been used in the endovascular treatment of descending thoracic aneurysms, ${ }^{5}$ solves this problem by providing a long, straight attachment area between the stent-graft and the intima of the true lumen. This method promotes less invasive therapy for acute type B aortic dissection and enables us to prevent the enlargement of the false lumen in the chronic phase. We believe that this method is effective enough to be generally recommended for cases in which the entry site formed near the left subclavian artery.

\section{REFERENCES}

1. Kato M, Matsuda T, Kaneko M, Kuratani T, Mizushima T, Seo Y, et al. Outcomes of stent-graft treatment of false lumen in aortic dissection. Circulation 1998;98(Suppl):II-305-12.

2. Nienaber CA, Fattori R, Lund G, Dieckmann C, Wolf W, Kodolitsch YV, et al. Nonsurgical reconstruction of thoracic aortic dissection by stent-graft placement. N Engl J Med 1999;340:1539-45.

3. Dake MD, Kato N, Mitchell RS, Semba CP, Razavi MK, Shimono T, et al. Endovascular stent-graft placement for the treatment of acute aortic dissection. N Engl J Med 1999;340:1546-52.

4. Kato M, Ohnishi K, Kaneko M, Ueda T, Kishi D, Mizushima T, et al. Determining surgical indications for acute type B dissection based on enlargement of aortic diameter during the chronic phase. Circulation 1995;92(Suppl):II-107-12.

5. Dake MD, Miller DC, Mitchell RS, Semba CP, Moore KA, Sakai T. The "first generation" of endovascular stent-graft for patients with aneurysms of the descending thoracic aorta. J Thorac Cardiovasc Surg 1998;116:689-704. 